\title{
The prevalence of autoimmune thyroiditis in adolescent girls with polycystic ovary syndrome
}

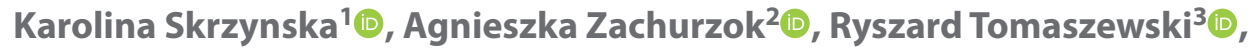 \\ Aneta Gawlik' ${ }^{1}$, Ewa Malecka-Tendera' ${ }^{10}$ \\ ${ }^{1}$ Department of Pediatrics and Pediatric Endocrinology, Faculty of Medical Sciences in Katowice, \\ Medical University of Silesia, Katowice, Poland \\ ${ }^{2}$ Department of Pediatrics, Faculty of Medical Sciences in Zabrze, Medical University of Silesia in Katowice, Poland \\ ${ }^{3}$ Department of Pediatric Traumatology and Orthopedics, Upper Silesian Children's Health Centre, Katowice, Poland
}

\begin{abstract}
Objectives: Both polycystic ovary syndrome (PCOS) and autoimmune thyroiditis (AT) are reported to be common endocrinopathies. In recent years the number of publications assessing the coexistence of these two disease entities in adult women has been growing. There are many suggestions regarding pathophysiological mechanisms that can cause the relationship between AT and PCOS. However, there is still a lack of research among adolescent girls.

The aim of the study was to analyze the occurrence of autoimmune thyroiditis in adolescent girls with PCOS.

Material and methods: The study group included 80 girls diagnosed with PCOS (chronological age: $16.54 \pm 1.00$ years, BMI: $22.80 \pm 3.27 \mathrm{~kg} / \mathrm{m}^{2}$ ), and the control group -64 regularly menstruating girls (chronological age: $16.71 \pm 0.63$ years, BMl: $24.8 \pm 5.2 \mathrm{~kg} / \mathrm{m}^{2}$ ). The thyroid function and morphology were assessed based on the concentration of thyroid stimulating hormone (TSH), free thyroxine (fT4), anti-thyroid peroxidase (anti-TPO), anti-thyroglobulin (anti-TG) antibodies and ultrasound scan of the thyroid gland.

Results: AT was diagnosed in 18 (22.5\%) girls from the study group and nine (14.06\%) from the control group ( $p>0.05)$. Positive anti-TPO titer was observed more often in the study group [21 patients (26.25\%)] than in the control group [9 girls (14.06\%)] ( $p=0.054)$. Moreover, an abnormal ultrasound scan of the thyroid gland characteristic for AT was found in 18 girls from the study group (22.50\%) and 8 girls from the control group $(12.50 \%)(p>0.05)$.

Conclusions: The results of the analyzed studies do not confirm a significant relationship between PCOS and AT in adolescent girls. However, in the group of girls with PCOS, autoimmune process exponents were more frequent (anti-TPO), reaching the borderline level of statistical significance.
\end{abstract}

Key words: polycystic ovary syndrome; autoimmune thyroiditis; adolescent girls

Ginekologia Polska 2022; 93, 12: 948-953

\section{INTRODUCTION}

Polycystic ovary syndrome (PCOS) is considered one of the most common endocrinopathy in women of reproductive age, and depending on the diagnostic criteria adopted, may affect $6-15 \%$ of women [1]. In the literature, we can find very divergent data on the epidemiology of PCOS in adolescents most likely due to the frequently modified, non-uniform PCOS criteria in adolescent girls and the large diversity of the group. It is estimated that PCOS may affect 0.56$3.1 \%$ of adolescent girls (based on the scarce data) $[2,3]$. While the diagnosis of PCOS among adult women is wide- spread and established, the subject should be approached with some caution among adolescent girls. Diagnosis in this age group is difficult and often requires long-term follow-up because what is considered pathology in adult women may not always be pathology in adolescent girls.

Thyroid dysfunction is also a common endocrinopathy and autoimmune thyroiditis (AT) comes to the fore in terms of its prevalence which may affect $5-20 \%$ of women of reproductive age and is the most common cause of hypothyroidism in women [4]. It is estimated that AT occurs in $0.3 \%-9.6 \%$ of children, and it affects girls more often

Corresponding author:

Karolina Skrzynska

Department of Pediatrics and Pediatric Endocrinology, Faculty of Medical Sciences in Katowice, Medical University of Silesia, 16 Medyków St, 40-752 Katowice, Poland e-mail: k.skrzynska@sum.edu.pl 
than boys, with a frequency ratio of even 4:1 to 8:1 depending on the ethnic group [5].

To date, several studies evaluating the relationship between AT and PCOS have been published. These studies were conducted on adult women. So far, no clear conclusions have been drawn and research on the search for pathophysiological pathways connecting these disease entities is still ongoing. The mechanisms responsible for the development of autoimmune thyroid processes are complex and include genetic and environmental factors, such as iodine intake in the diet, drugs, infections [6, 7]. Hormones also influence autoimmune processes. Similarly, among the etiological factors of PCOS, genetic factors are mentioned, as well as hormonal and metabolic factors [8-12]. Recently, the possible autoimmune basis of PCOS has been increasingly emphasized [13], which is confirmed by the published data describing the increased prevalence of AT among women with PCOS [14]. There are no reports analyzing the above relationship in the group of teenagers. For this reason, we undertook a study aimed at assessing the frequency of thyroid disorders, especially autoimmune thyroid disorders, in adolescent girls with PCOS.

\section{MATERIAL AND METHODS}

A total of 144 patients were qualified for the research. Eighty girls were included in the study group, while the remaining 64 regularly menstruating girls were included in the control group. The study group was previously described in Skrzynska et al. [15]. It included adolescent girls who, based on the conducted diagnostics, met all the criteria for the diagnosis of PCOS. The presence of the antibodies against thyroid peroxidase (anti-TPO) above $35 \mathrm{IU} / \mathrm{mL}$ and/or antibodies against thyroglobulin (anti-Tg) above $40 \mathrm{IU} / \mathrm{mL}$ with characteristic for AT thyroid image on USG was required to make a diagnosis of AT [16]. Abnormal thyroid function was not a necessary criterion to diagnose AT.

Both, in the study group and in the control group patients were qualified to participate in the study considering the following exclusion criteria: ij eating disorders of the anorexia nervosa type, bulimia;

ij hyperprolactinaemia [concentration of prolactin $(\mathrm{PRL}) \geq 36 \mu \mathrm{g} / \mathrm{L}]$;

ij adrenal cortex dysfunction;

ij decompensated, clinically significant disorders of the thyroid gland (TSH level $<0.4 \mathrm{mIU} / \mathrm{L}$ or $>10.0 \mathrm{mlU} / \mathrm{L}$ );

ij taking drugs within the last 3 months that could interfere with the determination of sex hormone levels.

Patients' data were analyzed: age [years], anthropometric measurements (body weight [kg], height [cm], BMI $\left.\left[\mathrm{kg} / \mathrm{m}^{2}\right]\right)$, gynecological age [years], age of menarche [years], hirsutism assessed according to the Ferriman-Gallwey scale [points], parameters of thyroid function (thyrotropic hormone - TSH [mlU/L], free thyroxine - fT4 [pmol/L], anti-TPO [IU/mL], anti-TG [IU/mL]), and the image of the thyroid gland. In the ultrasound examination using the Siemens Medical Solution USA, Inc. apparatus, equipped with the VFX 13-5 linear probe. As an image characteristic of autoimmune thyroiditis, we adopted the description of the heterogeneous, hypoechoic structure of the thyroid gland.

\section{Statistical analysis}

The obtained results were analyzed statistically using the Statistica 13.3 PL software. The quantitative variables were described with mean (standard deviation) and qualitative variables were defined by frequency (\%). Chi-square test for qualitative and Student's t test for quantitative data comparison of variables between the groups were used, as appropriate. A p value less than 0.05 was considered statistically.

The Bioethics Committee of the Medical University of Silesia approved the study (KNW/0022/KB/70/16). Considering the retrospective nature of the work, the Bioethics Committee did not require additional patient consents.

\section{RESULTS}

The clinical characteristics of the groups is presented in Table 1. No significant differences were found in chronological age, BMI and age of menarche between the groups

\begin{tabular}{|l|l|l|l|}
\hline \multicolumn{1}{|c|}{ Table 1. Comparison of the clinical and hormonal characteristics of the study and control groups } \\
\hline Variable & \multicolumn{1}{c|}{$\begin{array}{c}\text { Study group } \\
\mathbf{n = 8 0}\end{array}$} & $\begin{array}{c}\text { Control group } \\
\mathbf{n = 6 4}\end{array}$ \\
\hline Age [years] & $16.54 \pm 1.00$ & $16.71 \pm 0.63$ & $>0.05$ \\
\hline BMI [kg/m²] & $24.60 \pm 4.16$ & $22.80 \pm 3.27$ & $>0.05$ \\
\hline Age of menarche [years] & $12.00 \pm 1.00$ & $12.00 \pm 1.00$ & $>0.05$ \\
\hline Gynecological age [years] & $3.79 \pm 0.94$ & $4.50 \pm 0.92$ & 0.01 \\
\hline Hirsutism [points] & $6.47 \pm 6.00$ & $3.47 \pm 4.00$ & 0.003 \\
\hline Testosterone [nmol/L] & $2.11 \pm 0.70$ & $1.51 \pm 0.53$ & $<0.001$ \\
\hline
\end{tabular}

$\mathrm{BMI}$ - body mass index 


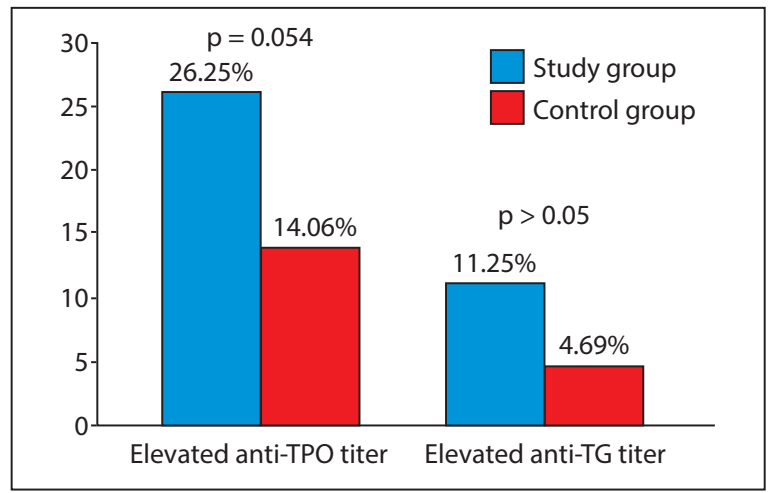

Figure 1. The incidence of positive anti-thyroid antibodies in the study and control groups

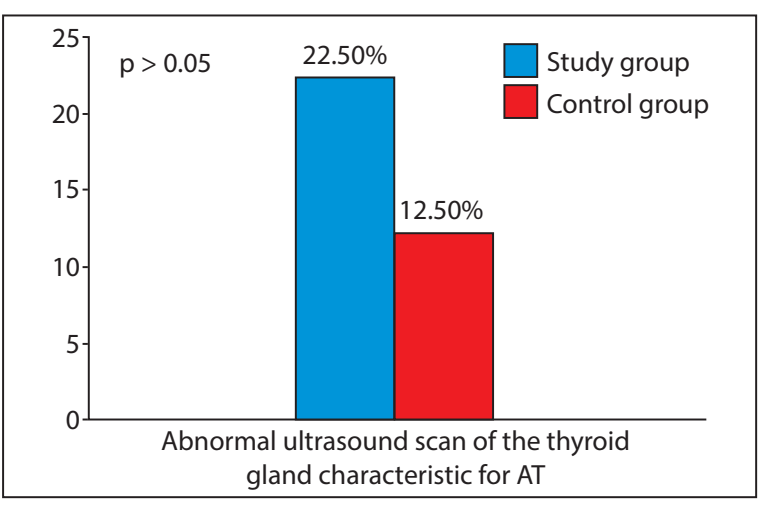

Figure 2. The frequency of occurrence of the image characteristic of AT in the ultrasound examination in the study and control group

$(p>0.05)$. However, it was shown that the gynecological age was significantly older in the control group than in the study group ( $p=0.01$ ). On the other hand, hirsutism score was significantly higher in the study group than in the control group ( $p=0.003$ ). Testosterone concentration in the study group was also higher than in the control group ( $<<0.001)$.

Elevated anti-TPO titer was found in 30 girls (20.83\%) in total. In the study group the increased titer of anti-TPO was reported in 21 patients (26.25\%) and in the control group — in 9 patients $(14.06 \%)(p=0.054)$. Moreover, 9 patients from the study group (11.25\%) had an increased titer of anti-TG, while in the control group there were 3 such patients (4.69\%) ( $p>0.05)$ (Fig. 1).

Eighteen girls from the study group (22.50\%) and eight girls from the control group (12.50\%) had an abnormal ultrasound scan of the thyroid gland characteristic for AT $(p>0.05)$ (Fig. 2). The difference in thyroid volume between the groups was also not significant ( $p>0.05$ ) and amounted to $8.7 \pm 3.4 \mathrm{~mL}$ in the control group and $9.2 \pm 4.0 \mathrm{~mL}$ in the study group.

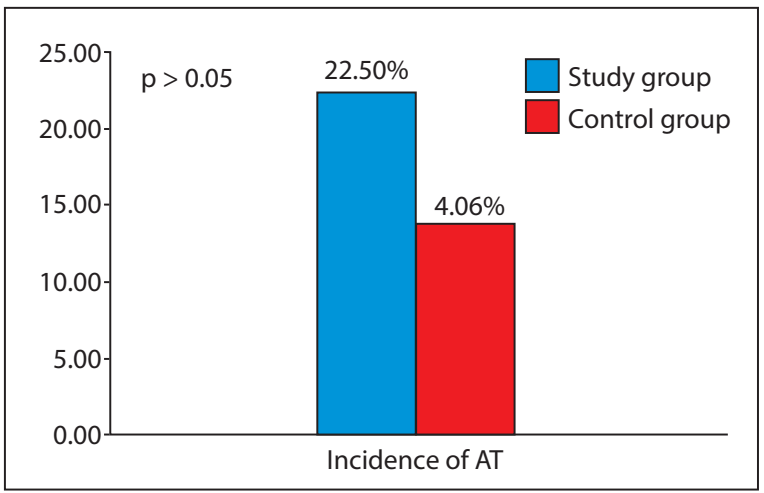

Figure 3. The frequency of AT diagnosis in accordance with the diagnostic criteria adopted in the study and control groups

A total of 27 patients (18.75\%) who participated in the study met the AT criteria. Among them the major part - 18 girls (22.50\%) came from the study group and 9 (14.06\%) from the control group. There was no significant difference in the incidence of AT between the groups ( $p>0.05$ ) (Fig. 3).

In the study group 7 patients (8.75\%) and in the control group $9(14.06 \%)$ had previously started treatment with levothyroxine due to hypothyroidism. Subclinical hypothyroidism $(\mathrm{SH})$ was diagnosed in a total of $6(4.17 \%)$ girls. None of the patients diagnosed with $\mathrm{SH}$ had previously been treated with levothyroxine. More patients with $\mathrm{SH}$ were recorded in the control group [4 girls (6.25\%)] than in the study group, where such a diagnosis was made only in 2 patients (2.50\%). What is more, the difference in the incidence of $\mathrm{SH}$ was not statistically significant $(p>0.05)$.

\section{DISCUSSION}

The aim of the study was to analyze the occurrence of thyroid dysfunction, especially AT, in girls with PCOS. The study group included 80 girls diagnosed with PCOS, and there were 64 girls with regular menses in the control group. AT was diagnosed in $22.5 \%$ of girls from the study group and $14.06 \%$ from the control group ( $p>0.05$ ). Positive anti-TPO antibody titer (26.25\%) was observed more often in the study group than in the control group (14.06\%) $(p=0.054)$. There was no significant difference in the incidence of thyroid dysfunction between the groups.

The incidence of thyroid diseases seems to be increased in women with PCOS what is suggested by published studies. Romitti et al. [17] in their meta-analysis conducted based on 13 studies showed significant relationship between AT and PCOS. It was found that AT is more common in women with PCOS than in the control group, regardless of geographic differences (OR $=3.27 ; 95 \% \mathrm{Cl} 2.32-4.63$ ). The prevalence of AT among women with PCOS in the compared studies 
ranged between $18.75 \%$ and $43.1 \%$, while in the control groups it ranged between $1.25 \%$ and $26.2 \%$. Only in 2 out of 13 studies the difference in the incidence of AT between women with PCOS and healthy women was not significant. The differences in the frequency of AT in individual studies are most likely resulted from the variability of the adopted AT diagnostic criteria between studies. What is more, Polish research also showed a relationship between AT and PCOS. The thyroid diseases were diagnosed in $31.3 \%$ of young women with PCOS and $70 \%$ of all thyroid diagnoses were AT, found in nearly $21 \%$ of the patients in the study group [18]. These results are similar to the results obtained in our study. We found AT in $22.5 \%$ of girls from the study group and $14.06 \%$ from the control group. However, these values did not differ significantly. One of the reasons for the lack of a clear difference between the groups may be characteristics of our study group different from adult women group. The course and the diagnostic criteria of PCOS in adolescent girls are different than in adult women. In addition, studies show that the prevalence of AT in women increases with age, which is not seen in men. AT may affect $5-20 \%$ of women of reproductive age and is the most common cause of hypothyroidism in women [19]. However, it is estimated that AT occurs only in $0.3 \%-9.6 \%$ of children in the pediatric population and is much more common in girls than in boys, with a frequency ratio of even 4:1 to 8:1 depending on the ethnic group [5]. The lack of significant difference between the prevalence of AT in the study group and the control group can arise from the young age of the patients. It cannot be ruled out that with age this difference would gain statistical significance also due to additional risk factors for autoimmune diseases resulting from the hormonal profile of women with PCOS and absent in healthy women. It is emphasized, that sex hormones (estrogens, progesterone and testosterone) have an impact on the functioning of the immune system. In women with PCOS an increased ratio of estradiol to progesterone might be involved in the stimulation of the immune system and consequently in the development of AT. The study involving population of adolescent gills with PCOS showed, that in the group of girls diagnosed with both PCOS and AT, the concentration of 2 2 was significantly higher than in the group of girls with PCOS but without AT ( $p=0.04)$. Additionally, a significant positive correlation between AT and estradiol concentration $\left(r_{y}=0.27 ; p=0.04\right)$ was found [15]. On the other hand, the lack of a clear difference in AT prevalence between the groups may also be related to the fact that both groups had a similar but high BMI. This seems to be an advantage of the study, because in this way the possible impact of obesity on the incidence of AT in the group of girls with PCOS was eliminated, as the latest research focuses on the relationship between BMI and the occurrence of AT.
The differences in the prevalence of AT among women with PCOS reported by different researchers might result not only from the PCOS diagnostic criteria used, but also from the diagnostic criteria for AT. Arduc et al. [20] obtained similar to ours the prevalence of AT in patients with PCOS, although the prevalence of AT in the control group differed between the work of Arduc et al. and ours [20]. They showed a significantly higher frequency of AT in women with PCOS (22.1\%) compared to the control group (5\%) ( $<<0.0004)$. The similarity of AT prevalence in the study group with the results of our study can be explained by the similar size of the groups, the young age of the patients and the same criteria for the diagnosis of AT, i.e., the presence of a positive titer of anti-TPO and/or anti-TG and an ultrasound image typical of chronic thyroiditis. In the study by Duran et al. [21] AT was diagnosed in as many as $32.5 \%$ of women with PCOS and in $23.3 \%$ in the control group. In order to diagnose AT, it was enough to meet only 1 of the 3 mentioned criteria, namely the presence of a positive titer of anti-TPO, a positive titer of anti-TG or the description of an abnormal ultrasound image characteristic of chronic thyroiditis.

Furthermore, numerous reports suggest that anti-TPO are claimed to be a more sensitive indicator of the autoimmune process in the thyroid gland than anti-TG. For this reason, some people refrain from determining the titer of anti-TG and use only anti-TPO in the diagnosis of AT. In our study, the frequency of positive anti-TPO titers in the study group was higher than in the control group $(26.25 \%$ and $14.06 \%$, respectively; $p=0.054)$. The frequency of elevated anti-TG titers was also higher in the study group, but it did not differ significantly between the groups ( $p>0.05$ ) and amounted to $11.25 \%$ in the study group and $4.69 \%$ in the control group. Al Saab et al. [22], analyzing 56 patients with PCOS, showed a positive titer of anti-TPO in $19.6 \%$ of them compared to $3.3 \%$ in the control group ( $p=0.037$ ) and a positive titer of anti-TG in $21.4 \%$ of women with PCOS and only $3.3 \%$ without diagnosed PCOS ( $p=0.026)$. On the other hand, in a study by Janssen et al. [23], a positive titer of anti-TPO was found in $26.9 \%$ of women with PCOS and only $8.3 \%$ of women in the control group. Menon et al. [24] also reported significantly more frequent occurrence of a positive titer of anti-TPO in women with PCOS (25\%) compared to $5 \%$ in the control group. The differences in the percentage distribution of the positive titer of anti-thyroid antibodies in the presented studies may result from the adopted cut-off points defining the positive titer of anti-TPO or anti-TG. It seems that geographical differences do not play a significant role in this case, which is confirmed by the published data [25-29]. Kim et al. [30] came to different conclusions regarding the distribution of anti-thyroid antibodies in women with PCOS. They described a positive titer of anti-TPO in $4.8 \%$ of the test group and $7.7 \%$ 
of the control group. Significant differences between the study cited and those described above may result from the analyzed population. In the study by Kim et al. [30], the patients were not hospitalized, but only assessed during follow-up visits to an outpatient health care center. Based on a systematic review of research and meta-analysis, it turns out that the phenotype of PCOS in patients diagnosed in a hospital environment differs compared to random populations [31]. Inpatients meet more PCOS diagnostic criteria and have a higher mean BMI than ambulatory patients. Therefore, it seems that the data of Kim et al. [30] come from an unselected group - difficult to reference and compare.

In our study, we did not find significant differences in the frequency of finding an abnormal image, characteristics of chronic thyroiditis on ultrasound, although in the study group the image typical for AT was found in $22.5 \%$ compared to $12.3 \%$ of girls from the control group. On the other hand, Janssen et al. [23] described a typical AT picture of the thyroid gland in $42.3 \%$ of patients in the PCOS group and in only $6.5 \%$ in the control group $(p<0.001)$. Similarly, Sinha et al. [34] showed a significantly higher percentage of the thyroid image typical for AT in the group of women with PCOS compared to the control group ( $12.5 \%$ vs $2.5 \%$, respectively). In both above-cited studies, no significant differences were found in the volume of the thyroid gland between the groups described. These evident differences in the percentage of patients with abnormal thyroid gland descriptions on ultrasound may be due to differences in BMI in women across studies. It turns out that the mean BMI of patients with PCOS in the work of Janssen et al. was $30.0 \pm 7.9 \mathrm{~kg} / \mathrm{m}^{2}$, and in the control group it was $25.5 \pm 7.1 \mathrm{~kg} / \mathrm{m}^{2}$ [23]. In the study by Sinha et al. [32], the mean BMI of women in the study group was significantly lower and amounted to $24.68 \pm 3.07 \mathrm{~kg} / \mathrm{m}^{2}$, and in the control group $23.55 \pm 3.02 \mathrm{~kg} / \mathrm{m}^{2}$. Some studies suggest that a significant degree of obesity (so-called morbid obesity, $\mathrm{BMI}>40 \mathrm{~kg} / \mathrm{m}^{2}$ ) may affect the morphology of the thyroid gland, i.e., its hypoechoic nature in ultrasound. It is a very good and well-known parameter helpful in the diagnosis of AT but it turns out to be a poor diagnostic parameter in patients with morbid obesity.

\section{CONCLUSIONS}

There was no significant difference in the prevalence of AT between the groups but in the group of girls with PCOS autoimmune process exponents were more frequent (anti-TPO), reaching the borderline level of statistical significance. Considering the young age of the patients and the tendency to increase the incidence of AT with age, it can be assumed that the differences in the incidence of AT may reach the level of statistical significance at a later age.

\section{Conflict of interest}

All authors declare no conflict of interest.

\section{REFERENCES}

1. Fauser BC, Tarlatzis BC, Rebar RW, et al. Consensus on women's health aspects of polycystic ovary syndrome (PCOS): the Amsterdam ESHRE/ASRM-sponsored 3rd PCOS Consensus Workshop Group. Fertil Steril. 2012; 97(1): 28-38.e25, doi: 10.1016/j.fertnstert.2011.09.024, indexed in Pubmed: 22153789.

2. Christensen SB, Black MH, Smith $\mathrm{N}$, et al. Prevalence of polycystic ovary syndrome in adolescents. Fertil Steril. 2013; 100(2): 470-477, doi: 10.1016/j.fertnstert.2013.04.001, indexed in Pubmed: 23756098.

3. SharifE, Rahman S, Zia Y, et al. The frequency of polycystic ovary syndrome in young reproductive females in Qatar. Int JWomens Health. 2017; 9: 1-10, doi: 10.2147/JJWH.S120027, indexed in Pubmed: 28031728.

4. Medici M, Porcu E, Pistis G, et al. Identification of novel genetic Loci associated with thyroid peroxidase antibodies and clinical thyroid disease. PLoS Genet. 2014; 10(2): e1004123, doi: 10.1371/journal.pgen.1004123, indexed in Pubmed: 24586183.

5. Zois C, Stavrou I, Kalogera C, et al. High prevalence of autoimmune thyroiditis in school children after alimination iodine deficiency in northwestern Greece. Thyroid. 2003; 13(4): 485-489, doi: 10.1089/105072503322021151, indexed in Pubmed: 12855016.

6. Mincer DL, Jialal I. Hashimoto Thyroiditis. StatPearls Publishing, Treasure Island (FL) 2020.

7. Mizukami Y, Michigishi T, Kawato M, et al. Chronic thyroiditis: thyroid function and histologic correlations in 601 cases. Hum Pathol. 1992; 23(9): 980-988, doi: 10.1016/0046-8177(92)90258-5, indexed in Pubmed: 1516932.

8. Kosova G, Urbanek M. Genetics of the polycystic ovary syndrome. Mo Cell Endocrinol. 2013; 373(1-2): 29-38, doi: 10.1016/j.mce.2012.10.009, indexed in Pubmed: 23079471.

9. Legro RS, Driscoll D, Strauss JF, et al. Evidence for a genetic basis for hyperandrogenemia in polycystic ovary syndrome. Proc Natl Acad Sci U S A. 1998; 95(25): 14956-14960, doi: 10.1073/pnas.95.25.14956, indexed in Pubmed: 9843997.

10. Kahsar-Miller MD, Nixon C, Boots LR, et al. Prevalence of polycystic ovary syndrome (PCOS) in first-degree relatives of patients with PCOS. Fertil Steril. 2001; 75(1): 53-58, doi: 10.1016/s0015-0282(00)01662-9, indexed in Pubmed: 11163816.

11. Drosdzol-Cop A, Tymińska-Bandoła A, Bil A, et al. Zespół policystycznych jajników u nastolatek - diagnostyka i leczenie. Ginekologia po Dyplomie. 2017(3).

12. Jakubowski L. Genetic aspects of Polycystic Ovary Syndrome [in Polish]. Endokrynol Pol. 2005; 56(3): 285-293, indexed in Pubmed: 16350721.

13. Hefler-Frischmuth K, Walch K, Huebl W, et al. Serologic markers of autoimmunity in women with polycystic ovary syndrome. Fertil Steril. 2010; 93(7): 2291-2294, doi: 10.1016/j.fertnstert.2009.01.056, indexed in Pubmed: 19296936.

14. Gaberšček S, Zaletel K, Schwetz V, et al. Mechanisms in endocrinology: thyroid and polycystic ovary syndrome. Eur J Endocrinol. 2015; 172(1): R9-21, doi: 10.1530/EJE-14-0295, indexed in Pubmed: 25422352.

15. Skrzyńska KJ, Zachurzok A, Gawlik AM. Metabolic and hormonal profile of adolescent girls with polycystic ovary syndrome with concomitant autoimmune thyroiditis. Front Endocrinol (Lausanne). 2021; 12: 708910, doi: 10.3389/fendo.2021.708910, indexed in Pubmed: 34276569.

16. Szczeklik A. Interna Szczeklika. Podręcznik chorób wewnętrznych. Medycyna Praktyczna, Kraków 2017: 1316-1317.

17. Romitti M, Fabris VC, Ziegelmann PK, et al. Association between PCOS and autoimmune thyroid disease: a systematic review and meta-analysis. Endocr Connect. 2018; 7(11): 1158-1167, doi: 10.1530/EC-18-0309, indexed in Pubmed: 30352422.

18. Szafraniec A, Porada D, Lenart-Lipińska M, et al. Częstość występowania chorób tarczycy u pacjentek zzespołem policystycznych jajników. Forum Med Rodz. 2016; 10(3): 140-144.

19. Medici M, Porcu E, Pistis G, et al. Identification of novel genetic Loci associated with thyroid peroxidase antibodies and clinical thyroid disease. PLoS Genet. 2014; 10(2): e1004123, doi: 10.1371/journal.pgen.1004123, indexed in Pubmed: 24586183.

20. Arduc A, Aycicek Dogan B, Bilmez S, et al. High prevalence of Hashimoto's thyroiditis in patients with polycystic ovary syndrome: does the imbalance between estradiol and progesterone play a role? Endocr Res. 2015; 
40(4): 204-210, doi: 10.3109/07435800.2015.1015730, indexed in Pubmed: 25822940.

21. Duran C, Basaran M, Kutlu O, et al. Frequency of nodular goiter and autoimmune thyroid disease in patients with polycystic ovary syndrome. Endocrine. 2015; 49(2): 464-469, doi: 10.1007/s12020-014-0504-7, indexed in Pubmed: 25522724.

22. Al-Saab R, Haddad S. Detection of thyroid autoimmunity markers in euthyroid women with polycystic ovary syndrome: a case-control study from syria. Int J Endocrinol Metab. 2014; 12(3): e17954, doi: 10.5812/ijem.17954, indexed in Pubmed: 25237328.

23. Janssen $\mathrm{OE}$, Mehlmauer N, Hahn S, et al. High prevalence of autoimmune thyroiditis in patients with polycystic ovary syndrome. Eur J Endocrinol. 2004; 150(3): 363-369, doi: 10.1530/eje.0.1500363, indexed in Pubmed: 15012623.

24. Menon M, Ramachandran V. Antithyroid peroxidase antibodies in women with polycystic ovary syndrome. J Obstet Gynaecol India. 2017;67(1): 61-65, doi: 10.1007/s13224-016-0914-y, indexed in Pubmed: 28242970.

25. Janovsky CC, Bittencourt MS, Goulart AC, et al. Prevalence of antithyroperoxidase antibodies in a multiethnic Brazilian population: the ELSA-Brasil Study. Arch Endocrinol Metab. 2019; 63(4): 351-357, doi: 10.20945/2359-3997000000122, indexed in Pubmed: 31038589.

26. O'Leary PC, Feddema PH, Michelangeli VP, et al. Investigations of thyroid hormones and antibodies based on a community health survey: the Busselton thyroid study. Clin Endocrinol (Oxf). 2006; 64(1): 97-104, doi: 10.1111/j.1365-2265.2005.02424.x, indexed in Pubmed: 16402936.
27. Kasagi K, Takahashi N, Inoue G, et al. Thyroid function in Japanese adults as assessed by a general health checkup system in relation with thyroid-related antibodies and other clinical parameters. Thyroid. 2009; 19(9): 937-944, doi: 10.1089/thy.2009.0205, indexed in Pubmed: 19678737.

28. Unnikrishnan AG, Kalra S, Sahay RK, et al. Prevalence of hypothyroidism in adults: An epidemiological study in eight cities of India. Indian J Endocrinol Metab. 2013; 17(4): 647-652, doi: 10.4103/2230-8210.113755, indexed in Pubmed: 23961480.

29. Pedersen IB, Knudsen N, Jørgensen T, et al. Thyroid peroxidase and thyroglobulin autoantibodies in a large survey of populations with mild and moderate iodine deficiency. Clin Endocrinol (Oxf). 2003; 58(1): 36-42, doi: 10.1046/j.1365-2265.2003.01633.x, indexed in Pubmed: 12519410.

30. Kim JJu, Yoon JiW, Kim MJ, et al. Thyroid autoimmunity markers in women with polycystic ovary syndrome and controls. Hum Fertil (Camb). 2020 [Epub ahead of print]: 1-7, doi: 10.1080/14647273.2019.1709668, indexed in Pubmed: 31910041.

31. Lizneva D, Kirubakaran R, Mykhalchenko K, et al. Phenotypes and body mass in women with polycystic ovary syndrome identified in referral versus unselected populations: systematic review and meta-analysis. Fertil Steril. 2016; 106(6): 1510-1520.e2, doi: 10.1016/j.fertnstert.2016.07.1121, indexed in Pubmed: 27530062.

32. Sinha U, Sinharay K, Saha S, et al. Thyroid disorders in polycystic ovarian syndrome subjects: a tertiary hospital based cross-sectional study from Eastern India. Indian J Endocrinol Metab. 2013; 17(2): 304-309, doi: 10.4103/2230-8210.109714, indexed in Pubmed: 23776908. 